\title{
Sutura arterial com técnicas contínua e de pontos separados, utilizando-se os fios polipropilene e polidioxanone. Estudo experimental em coelhos ${ }^{1}$
}

\author{
Nelson Leonardo Kerdahi Leite de Campos² \\ Anete Kinumi Ueda ${ }^{3}$ \\ Marcos Augusto de Moraes-Silva ${ }^{4}$
}

\begin{abstract}
Campos NLKL, Ueda AK, Moraes-Silva MA. Sutura arterial com técnicas contínua e de pontos separados, utilizando-se os fios polipropilene e polidioxanone: estudo experimental em coelhos. Acta Cir Bras [serial online] 2003 Set-Out;18(5). Disponível em URL: http://www.scielo.br/acb.

RESUMO - Objetivo: Observar o comportamento da sutura arterial em aortas abdominais de coelhos em crescimento, comparando-se as técnicas contínua e com pontos separados, empregandose dois tipos de fios: Polipropilene 7-0 (inabsorvível) e Polidioxanone 7-0 (absorvível). Métodos: Grupos: GI - Controle (sem sutura); GII - Polipropilene, Pontos Separados; GIII - Polipropilene, Contínua; GIV - Polidioxanone, Pontos Separados e GV - Polidioxanone, Contínua. Cada grupo foi subdividido em quatro Momentos de Eutanásia: aos 7, 14, 30 e 60 dias de pós-operatório. Foram avaliados: peso dos animais, diâmetros e pulsos arteriais, estenose, trombose, aderências, aortografia, visibilidade do fio, cicatrização e microscopia. Resultados: a) após 60 dias, o local da linha de sutura cresceu de forma significativa em todos os grupos; b) a técnica de sutura com pontos separados causou menor estenose da linha de sutura, observada tanto no ato cirúrgico, como na eutanásia dos animais; c) no exame histopatológico, as diferenças encontradas entre grupos foram transitórias, não persistindo após 60 dias de pós-operatório. Conclusão: O polidioxanone mostrou ser a melhor opção, entre os dois fios, para sutura de artérias em crescimento, pois causa pouca ou nenhuma restrição ao crescimento arterial na linha de sutura, mesmo quando se emprega a técnica contínua.
\end{abstract}

DESCRITORES - Artéria. Sutura. Polipropilene. Polidioxanone.

\section{Introdução}

Alguns autores afirmam que as técnicas cirúrgicas contínuas e de pontos separados em sutura arterial seriam equivalentes em seu resultado final, contudo a técnica contínua apresentaria discreta tendência à estenose e se houvesse ruptura do fio, ocorreria deiscência total da sutura ${ }^{10}$. Para outros autores, a técnica contínua seria de maior rapidez para sua execução e causaria menor número de perfurações na parede do vaso ${ }^{5}$.
Em se tratando de vasos em crescimento, Johnson e col. ${ }^{4}$ afirmam que a sutura arterial com técnica contínua em toda circunferência do vaso, impediria o crescimento do mesmo, ao menos que o fio se rompa; propõe então, para estes casos, a sutura com técnica contínua em parede posterior do vaso e de pontos separados na anterior. Para Pae e col. ${ }^{9}$, mesmo esta técnica levaria até $33 \%$ de reestenose nas crianças submetidas à cirurgia de correção de coarctação de aorta. A utilização de fios absorvíveis, surge como alternativa para sutura de vasos em crescimento; entre

1. Resumo de Tese de Mestrado do Curso de Pós - Graduação em Bases Gerais de Cirurgia e Cirurgia Experimental da Faculdade de Medicina de Botucatu, da Universidade Estadual Paulista (UNESP).

2. Médico da Disciplina de Cirurgia Cardiovascular da Faculdade de Medicina de Botucatu-UNESP. Mestre em Cirurgia.

3. Professora Assistente, Doutora do Departamento de Patologia da Faculdade de Medicina de Botucatu - UNESP.

4. Professor Assistente Doutor do Departamento de Cirurgia e Ortopedia da Faculdade de Medicina de Botucatu - UNESP. Responsável pela Disciplina da Cirurgia Cardiovascular. 
estes fios, o monofilamentar polidioxanone, possui alta resistência à força tensil, é absorvido por hidrólise, causa baixa lesão tecidual, sendo de fácil manuseio e é totalmente absorvido após 6 meses. Segundo Myers e col. ${ }^{6}$, este seria o melhor fio para emprego em cirurgias cardíacas pediátricas.

Apesar de relatos de sucesso no uso em cirurgia vascular, os fios absorvíves são relativamente pouco utilizados, provavelmente, entre outras razões, pelo temor que estes materiais venham a ser absorvidos antes da completa cicatrização da linha de sutura nos vasos.

Procuramos neste estudo experimental, comparar o uso de fios absorvíveis (polidioxanone) e inabsorvíveis (polipropilene), nas técnicas de sutura contínua e de pontos separados em aortas abdominais de coelhos em crescimento.

\section{Métodos}

Neste trabalho foram utilizados coelhos machos da raça Nortfolk com peso variando entre $1500 \mathrm{~g}$ e 2560g. Grupos: Grupo I (GI): Grupo Controle- 12 animais submetidos à exposição e isolamento da aorta abdominal; Grupo II (GII): 14 animais, submetidos à sutura da aorta abdominal com pontos separados, utilizando-se o fio sintético, inabsorvível, monofilamentar, Polipropilene 7-0; Grupo III (GIII): 12 animais submetidos à sutura contínua (chuleio) da aorta abdominal, com fio Polipropilene 7-0; Grupo IV (GIV): 13 animais, submetidos à sutura de aorta abdominal com pontos separados, utilizando-se o fio sintético, absorvível, momofilamentar, Polidioxanone7-0; GrupoV (GV) : 13 animais submetidos à sutura contínua (chuleio) de aorta abdominal, com fio Polidioxanone 7-0.

Os animais foram distribuidos por sorteio, nos diferentes grupos. Todos procedimentos cirúrgicos, medidas, avaliações (exceto microscopia) foram realizados pelo mesmo indivíduo (autor).

Técnica cirúrgica - Foi feita epilação e punção venosa na orelha direita. Para anestesia utilizou-se Pentobarbital sódico, na dose de $30 \mathrm{mg}$ por quilo, mantendo hidratação parenteral com solução de cloreto de sódio a $0,9 \%$ com glicose durante todo procedimento cirúrgico e recuperação pós-anestésica, na dosagem de 20 a $30 \mathrm{ml}$ por quilo de peso por hora.

Os procedimentos cirúrgicos foram realizados com técnica asséptica e uso amplificação através de lupa com aumento de duas vezes. O animal foi submetido à laparotomia logo abaixo do diafragma, até a altura da bexiga, com exposição da aorta abdominal transperitonial, abaixo de emergência das artérias renais. $\mathrm{O}$ diâmetro arterial foi medido $2 \mathrm{~cm}$ abaixo das artérias renais e avaliou-se o pulso arterial pela palpação. A seguir, procedeu-se, a heparinização endovenosa (100u de heparina), isolamento dos ramos arteriais abaixo das artérias renais, com dupla laçadura. Pinçamento da aorta proximal (1 $\mathrm{cm}$ abaixo das artérias renais) e distal, ( $3 \mathrm{~cm}$ abaixo). Nos animais a serem submetidos à sutura , realizou-se incisão na parede anterior da aorta, $1,5 \mathrm{~cm}$ abaixo da pinça proximal, sendo medida a extensão deste corte. As porções distais e proximais da artéria foram lavadas com solução heparinizada. Sutura com pontos separados: passagem de um ponto em cada ângulo do corte arterial que foram reparados; a seguir foi dado um ponto no centro do corte e um ponto entre o ponto central e cada ângulo (total de cinco pontos). Sutura contínua: Passagem de um ponto nos ângulos do corte, amarrando o fio, em seguida foi reparada uma das extremidades e com a outra, realizada sutura contínua , em chuleio, a partir dos ângulos, em direção ao centro da incisão. Após o término da sutura, foram feitas as medidas dos diâmetros da aorta nas localizações, pré, pós e na linha de sutura Também foi feita avaliação do pulso arterial, pré e pós linha de sutura; da presença de estenose na linha de sutura; da visibilidade do fio e da própria linha de sutura. No grupo controle, foram medidos os diâmetros da aorta, avaliados o pulso e a presença de estenose nos locais correspondentes aos dos grupos onde a aorta foi suturada.

Momentos de eutanásia - M7, M14, M30 e M60:

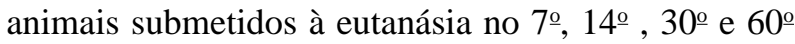
dia de pós-operatório, respectivamente.

Aortografia - Na eutanásia, foi realizada laparotomia, a aorta era isolada, fixou-se um alfinete ao lado da sutura para marcação deste local. Feita injeção de 4 $\mathrm{ml}$ de contraste Hexabrix na porção proximal da aorta realizando-se as radiografias (aparelho de raiox portátil Spectro II-Atlante, filmes Kodak 18/24, distância entre o animal e o colimador de $80 \mathrm{~cm}$, aplicação de $65 \mathrm{KV}$ em tempo de 0,12 segundos, a revalação das radiografias foi pelo processo manual).

Eutanásia - Os animais foram submetidos à eutanásia na data prevista, e realizadas as mesmas medidas e avaliações feitas no pós-operatório imediato.

Após retirada da porção da aorta, a eutanásia foi feita por embolia aérea. Na aorta retirada foi feita incisão na face oposta ao local da sutura (e nos locais correspondentes nas artérias do grupo controle, onde a sutura não ocorreu), expondo-se toda porção interna da artéria, fotografadas as peças, procedendo-se, então o estudo macro e microscópico.

Estudo anatomopatológico - Macroscopia: visibilidade do fio e da sutura, presença de estenose e trombos. Microscopia: após um período de fixação de no mínimo 48 horas, o fragmento de aorta foi 
seccionado longitudinalmente, no sentido transversal à sutura, foram obtidas, então duas fitas de tecido membranáceo, as quais foram incluidas em parafina e cortadas no micrótomo comum, em secções de 4 a 5 micra. As secções histológicas foram coradas pelas técnicas de Hematoxilina-Eosina, Alcian blue ph 2,5 e Calleja modificado, sendo então fotografadas.

Análise estatística dos resultados - As variáveis: peso inicial e na eutanásia, ganho de peso, diâmetro arterial externo antes da sutura, tamanho da incisão na artéria, diâmetro arterial proximal, no local e distal à sutura, aferidos logo após a realização desta e na eutanásia do animal, e aumento do diâmetro da aorta entre a cirurgia e a eutanásia, nas posições proximal, no local e distal à sutura, foram estudadas por meio de Análise de Variância para experimento inteiramente casualizado, cujos tratamentos são a combinação do tipo de fio (polipropilene e polidioxanone) e a técnica de sutura empregada (pontos separados e contínua), acrescido do grupo Controle. A avaliação foi executada em quatro momentos de eutanásia: M (M7, M14, M30 e M60).

A constituição das combinações dos fatores resultou no esquema fatorial $5 \times 4$ (cinco grupos em quatro modelos), com exceção da variável tamanho da incisão arterial, onde o grupo Controle foi descartado, tornando-se, portanto, um esquema 4 x 4 (quatro grupos e quatro momentos). As médias foram comparadas duas a duas, dentro dos grupos e momentos, pelo Teste de Tukey.

Todas as conclusões referentes à estatística foram efetuadas ao nível de $5 \%$ de significância.

As demais variáveis envolvidas no presente trabalho, foram discutidas através de estatística descritiva das freqüências percentuais de ocorrência.

\section{Resultados}

Houve ganho de peso significativo e igual, após 2 meses de cirurgia (M60), em todos os grupos. Em cada um dos outros momentos estudados (M7, M15 e M30) o perfil de ganho de peso também foi igual na comparação entre os grupos.

O diâmetro da artéria na linha de sutura mostrou o mesmo comportamento do ganho de peso, isto é, aumentou significativamente do momento da cirurgia até M60, de forma igual em todos os grupos (Tabela 1).

TABELA 1 - Média, desvio padrão e resultado do teste estatístico da comparação entre os aumentos dos diâmetros arteriais externos $(\mathrm{mm})$ nas linhas de sutura, no período entre a intervenção cirúrgica e o sacrifício, segundo grupos e momentos.

\begin{tabular}{lcccccccc}
\hline & \multicolumn{1}{c}{ M1 } & M2 & M3 & M4 \\
\hline G I & $0,00+0,00$ & $\mathrm{aA}$ & $0,20+0,00$ & $\mathrm{aA}$ & $0,40+0,35$ & $\mathrm{aA}$ & $0,93+0,12$ & $\mathrm{aB}$ \\
G II & $0,00+0,00$ & $\mathrm{aA}$ & $0,33+0,31$ & $\mathrm{aAB}$ & $0,50+0,12$ & $\mathrm{aB}$ & $0,50+0,35$ & $\mathrm{aB}$ \\
G III & $0,06+0,12$ & $\mathrm{aA}$ & $0,13+0,23$ & $\mathrm{aA}$ & $0,20+0,20$ & $\mathrm{aA}$ & $0,66+0,23$ & $\mathrm{aB}$ \\
G IV & $0,00+0,00$ & $\mathrm{aA}$ & $0,27+0,31$ & $\mathrm{aA}$ & $0,40+0,00$ & $\mathrm{aAB}$ & $0,80+0,20$ & $\mathrm{aB}$ \\
G V & $0,20+0,00$ & $\mathrm{aA}$ & $0,20+0,20$ & $\mathrm{aA}$ & $0,47+0,12$ & $\mathrm{aAB}$ & $0,90+0,35$ & $\mathrm{aB}$ \\
\hline
\end{tabular}

As letras minúsculas indicam as semelhanças ou diferenças estatisticamente significantes, entre os grupos dentro de um mesmo momento de eutanásia. As letras maiúsculas indicam as semelhanças ou diferenças estatisticamente significantes entre os momentos de eutanásia de um mesmo grupo.

Logo após o término da cirurgia, no GV (fio polidioxanone com técnica de sutura contínua), encontramos discreta diminuição do pulso arterial, palpável distalmente à sutura, de ++++ (antes da cirurgia) para +++ (após a cirurgia). No momento da eutanásia houve normalização do pulso que retornou à intensidade de ++++ .
Não foram encontradas diferenças relevantes entre os grupos quanto a presença de tecidos aderidos à linha de sutura.

No ato cirúrgico, ao término da sutura na aorta, em alguns grupos, a avaliação macroscópica da porção externa da linha de sutura mostrou aspecto estenótico. Isto ocorreu com maior freqüência nos grupos de técnica contínua, especialmente quando se utilizou o fio polipropilene (GIII). Nos grupos onde se utilizou a técnica com pontos separados, o aspecto estenótico foi pouco evidente com fio polipropilene (GII), e não ocorreu onde se utilizou o fio polidioxanone (GIV). 
Ao avaliarmos, na eutanásia dos animais a presença e intensidade de estenose da face externa da artéria, encontramos que esta foi também mais importante em GIII (polipropilene - sutura contínua), seguido dos grupos GII (polipropilene - pontos separados) e GV (polipropilene - sutura contínua), que se comportaram de forma semelhante. No GIV (polidioxanone pontos separados ) não ocorreu aspecto estenótico nas linhas de sutura. No GV (polidioxanone - sutura contínua) houve importante diminuição do grau de estenose em relação ao observado, imediatamente após a sutura.

O aspecto macroscópico da linha de sutura, na face luminal do vaso, mostrou o mesmo comportamento quanto à presença de estenose ao observado na face externa da arteria, exceto pelo fato de termos encontrado estenose também em GIV, porém menor e menos freqüente que nos outros grupos submetidos à sutura.
O exame macroscópico, no momento da eutanásia, mostrou que a visibilidade do fio na linha de sutura, nas faces externa e luminal do vaso, foi maior nos grupos que utilizaram fio polipropilene (GII e GIII), em relação aos grupos do fio polidioxanone (GIV e $\mathrm{GV})$. Na face luminal da linha de sutura, foram encontrados pequenos coágulos em algumas artérias. Preferimos chamar de coágulos em lugar de trombos, pois não foi possível comprovar a existência de trombose junto à linha de sutura ao estudo histopatológico. A comparação entre os grupos não demonstrou diferença quanto à freqüência ou tamanho destes coágulos.

A presença de estenose à arteriografia (intensidade e frequiência) foi maior nos grupos com técnica contínua de sutura (Figura 1), sendo em GIII maior que GV. Dentre os grupos com técnica de pontos separados a estenose foi menos intensa e freqüente em GII.

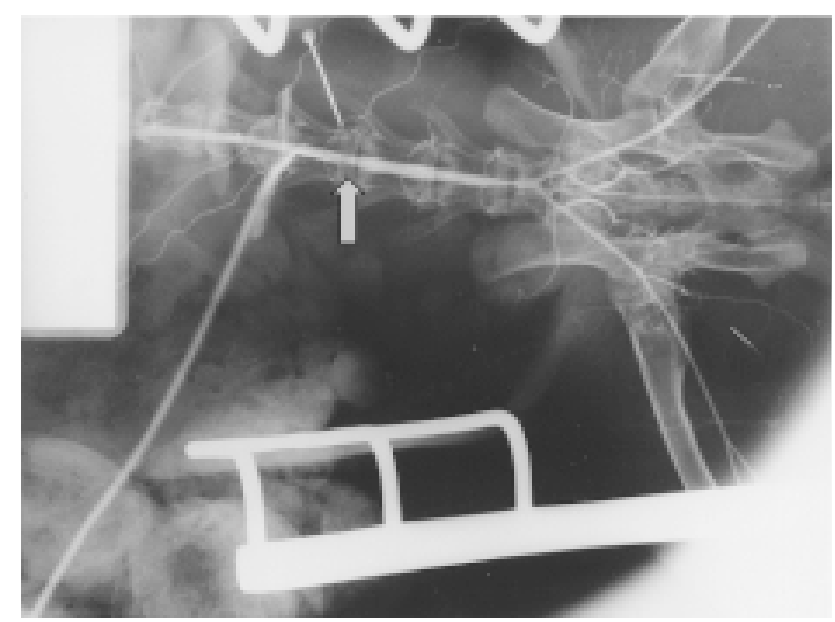

FIGURA 1 - Aortografia de animal do grupo GV - M2. Seta indica linha de sutura com estenose.

O estudo histopatológico avaliou, o grupo controle (GI) e os outros quatro grupos (GII a GV) em cada um dos momentos de sacrifício (M).

Grupo Controle (GI). Os animais do grupo controle apresentaram, na aorta, aspectos microscópicos semelhantes, em todos os momentos estudados (7, 14, 30 e 60 dias de P.O.), com características histológicas normais de artéria elástica:

Camada Íntima: geralmente delgada, revestida por camada única de células endoteliais, justapostas à membrana elástica interna, com escasso tecido conjuntivo subendotelial.

Camada Média: constituída por células musculares lisas, fibras colágenas e numerosas fibras elásticas; membrana elástica externa pouco evidente.
Camada Adventícia: constituída por tecido conjuntivo denso, acompanhado pordiscreta a moderada quantidade de fibras elásticas.

Além dos aspectos normais acima referidos, em 4 animais (um em cada momento), observou-se também, na superfície externa da camada adventícia (tecidos moles peri-adventiciais), a presença de processo inflamatório granulomatoso, tipo corpo estranho, com estruturas refringentes, pequenas, arredondadas ou alongadas, compatíveis com pêlos dos animais.

\section{Grupos com sutura (GII, GIII, GIV e GV):}

M 7 (7 dias de P.O.).Os quadros morfológicos foram semelhantes nos quatro grupos (GII, GIII, GIV e GV). Nos locais dos fios de sutura, notou-se tecido 
necrótico adjacente, intensa proliferação de fibroblastos, de fibras colágenas e discreta a moderada reação inflamatória linfo-mononuclear. Processo granulomatoso de discreta intensidade, ao redor do fio de sutura, estava presente em raros animais, sendo sempre mais evidente, quando havia presença de pêlos.

Nos cortes histológicos, onde os fios de sutura estavam presentes na superfície luminal do vaso, esta se mostrou irregular, com saliências ou reentrâncias, com grumos de tecido necrótico, constituídos por emaranhados de fibras musculares lisas, colágenas e principalmente elásticas. Foi também observada neoformação colágena, na camada íntima.

M 14 (14 dias de P.O.). A maioria dos aspectos histológicos foram semelhantes entre os quatro grupos . As diferenças observadas foram : a) focos de calcificação nas camadas média e adventícia, mais freqüentes e intensos no grupo GII (polipropilene - sutura contínua); b) hiperplasia da camada íntima mais evidente nos grupos GIV e GV (ambos com uso de polidioxanone).

Em relação ao M1, em M2 notou-se, ainda, a presença de material necrótico, junto ao fio de sutura; o processo inflamatório era mais discreto, sendo mais intensa a neoformação conjuntiva da íntima e adventícia.

M 30 (30 dias de P.O.).Aspectos histopatológicos semelhantes entre os grupos, com presença, em todos, de calcificação substituindo o material necrótico. Não houve diferença entre os grupos, quanto à hiperplasia da camada íntima. O processo inflamatório mononuclear ainda estava presente, sendo a fibrose mais madura e extensa.

M 60. (60 dias de P.O.). As semelhanças do quadro histológico foram mantidas na comparação entre os grupos: intensa hiperplasia conjuntiva da camada íntima, necrose com calcificação intensa e fibrose da adventícia. (Figura 2).

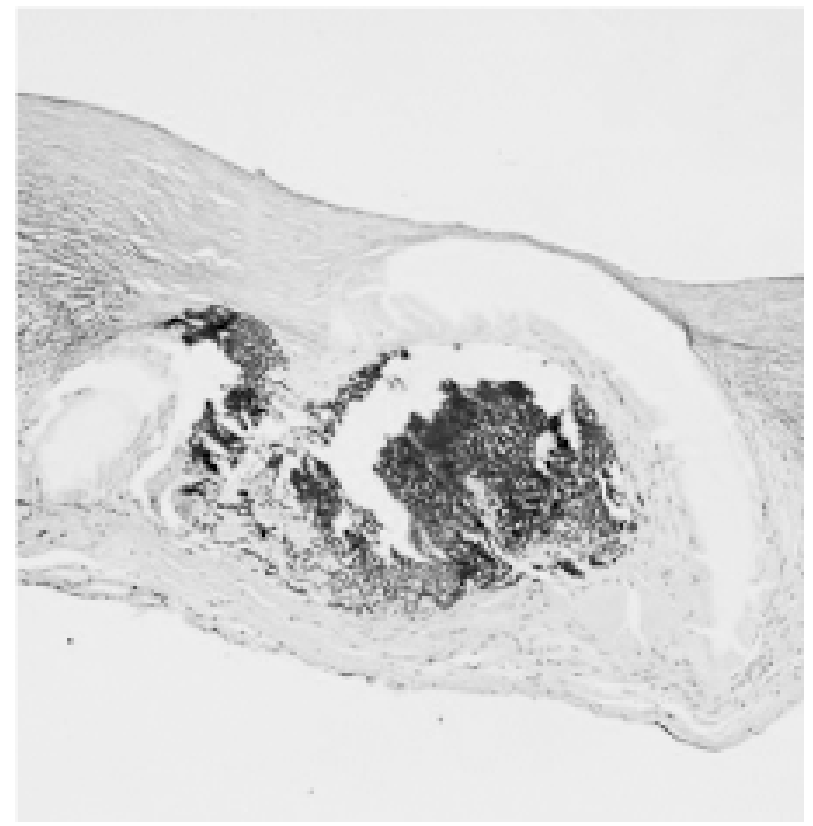

FIGURA 2 - Polidioxanone - Sutura contínua.

Fio de sutura em degeneração, corado em rosa na periferia. (Calleja, $25 \mathrm{X}$ - A.O.).

Luz Arterial (L). Local do fio de sutura (F).

Necrose com calcificação ( $\rightarrow$ ). Intensa fibrose da adventícia (A).

\section{Discussão}

A análise do ganho de peso mostrou o crescimento de todos animais e que este crescimento foi igual em todas grupos experimentais. Outros autores ${ }^{12}$, encontraram resultados semelhantes na comparação entre suturas arteriais com fios absorvíveis e inabsorvíveis
Preferimos estudar a sutura arterial limitando-se a metade da circunferência total do vaso, ficando preservado o restante deste. Este modo de agir, deveuse ao fato de que, na prática cirúrgica, constantemente são realizados procedimentos em artérias em crescimento, nas quais as suturas ocorrem somente em parte do perímetro total do vaso (ex: canulação de artéria 
femoral para perfusão temporária em circulação extracorpórea, com posterior sutura do vaso, suturas para lesões traumáticas em artérias, suturas em grandes vasos intratorácicos, etc.), a maioria dos trabalhos encontrados na literarura, no entanto utilizaram anastomoses término-terminais dos $\operatorname{vasos}^{1,2,3,4,7,7,9,9,11,12,13,14}$.

Observamos, como também relataram outros autores $^{7,11}$, que as características de uso na prática cirúrgica dos fios de polipropilene e polidioxanone são semelhantes: facilidade no manuseio geral e boa segurança na execução de nós.

Autores estudando tipos de anastomoses em artérias carótidas de cães ${ }^{10}$ e carótidas de ratos ${ }^{5}$, concluiram que a técnica contínua apresenta maior tendência de estenose da linha de sutura. Temos razão para concordar com estes estudos, pois em nosso trabalho, a técnica de sutura contínua com fio polipropilene promoveu, logo após o término da intervenção, menor diâmetro arterial externo, na linha de sutura, quando comparada com a técnica com pontos separados, utilizando-se o mesmo fio. Além disso, o pulso arterial, palpável logo após o término da sutura, apresentou diminuição de intensidade da posição pré para a pós sutura, somente em animais pertencentes aos grupos submetidos à sutura contínua . No momento do ato cirúrgico, o tipo do fio utilizado, não foi importante para o resultado final da sutura.

Após 60 dias de pós-operatório, nos grupos com sutura da aorta, o crescimento dos diâmetros arteriais, na linha de sutura foi significativo e igual, não importando, o fio ou a técnica empregados. Apesar de não se encontrarem diferenças significativas nas medidas dos diâmetros arteriais na linha de suturas, comparandose os grupos no sacrifício, notamos que o aspecto estenótico neste local foi diferente.

Estudos realizados em aorta de porcos em crescimento, comparando-se técnicas de sutura e fios inabsorvíveis (polipropilene) e absorvíveis (ácido poliglicólico), mostraram , após o crescimento arterial, que o único grupo onde a estenose não ocorreu foi o que empregou o fio absorvível, mesmo utilizando-se a técnica contínua ${ }^{9}$.

Em nosso experimento, após dois meses, encontramos diminuição do aspecto estenótico externo observado logo após o término da sutura, nos grupos onde se utilizou a técnica contínua e o fio polidioxanone. $\mathrm{Na}$ eutanásia dos animais, o exame da linha de sutura, na face luminal do vaso, e a arteriografia, mostraram menor grau de estenose nos grupos onde se empregou fio absorvível, e entre esses os com técnica de sutura com pontos separados. Estes dados podem sugerir, que em artérias em crescimento, mesmo quando se utiliza técnica de sutura contínua, pode-se obter resultados satisfatórios, isto é, pouca estenose da linha de sutura, se empregarmos o fio absorvível.

Nenhum dos animais estudados apresentou aumento de diâmetro ou dilatação aneurismática, na linha de sutura. Myers e col. ${ }^{6}$, avaliando a evolução de 46 cirurgias cardiovasculares em crianças, nas quais empregou o fio polidioxanone realizando arteriografias no pós-operatório, não observou a presença de formação aneurismática nas linhas de sutura. O surgimento de aneurismas não foram citados em outros estudos experimentais, envolvendo o fio de polidioxanone. . $^{8,11}$ Contudo, Verschure e col. ${ }^{14}$ encontraram dilatações aneurismáticas significativas na linha de sutura em aortas abdominais de porcos em crescimento, após 6 meses de cirurgia, usando fio polidioxanone com técnica de sutura contínua.

O estudo histopatológico, neste trabalho, teve por objetivo avaliar se o tipo de material do fio exerceria influência sobre o crescimento arterial na linha de sutura. Entretanto, as diferenças encontradas à microscopia foram discretas ou transitórias.

\section{Conclusões}

A técnica de sutura mais adequada foi com a pontos separados, por causar menor estenose da linha de sutura, observada tanto no ato cirúrgico, como no eutanásia dos animais. O polidioxanone mostrou ser a melhor opção, para sutura de artérias em crescimento, pois causa pouca ou nenhuma restrição ao crescimento arterial na linha de sutura, mesmo quando se emprega a técnica contínua.

\section{Referências}

1. Chiu I, Hung C, Chao S, Huang S, How S. Growth of the aortic anastomosis in pigs: comparision of continuos absorbable suture with nonabsorbable suture. J Thorac Cardiovasc Surg 1988; 95:112-8.

2. Dardik H, Dardik I, Katz A, Smith RB, Schwibner BH, Laufman $\mathrm{H}$. A new absorbable synthetyc suture in growing and adult primary anastosis: morphologic study. Surgery 1970; 68:1112-21.

3. Johnson J, Kirby CK. The relationship of the method of suture to the growth of end-to-end arterial anastomoses. Surgery 1950; 27: 17-25.

4. Johnson J, Kirby CK, Allam MW, Hagan W. The growth of vascular anastomoses with continuos posterior and interrupted anterior silk sutures. Surgery 1951; 29:721-5.

5. Little JR, Salerno TA. Contínuous suturing for microvascular anastomosis. J Neurosurg 1978; 48:1042-5.

6. Myers JL, Campbell DB, Waldhausen JA. The use of monofilament polydioxanone suture in pediatric cardio vascular surgery. J Thorac Cardiovasc Surg 1986;92:771-5.

7. Myers JL, Pae WE, Waldhausen JA, Pierce WS. Vascular anastomoses in growing vessels: comparission of absorbable 
polydioxanone and nonabsorbable polypropilene monofilament suture materials. Surg Forum 1981; 3: 339- 41.

8. Myers JL, Waldhausen JA, Pae WE, Abt AB, Prophet GA, Pierce WS. Vascular anastomoses in growing vassels: the use of absorbable sutures. Ann Thorac Surg 1982; 34: 529-37.

9. Pae WE, Waldhausen JA, Prophet GA, Pierce WS. Primary vascular anastomosis in growing pigs: comparison of polypropilene and polyglycolic acid sutures. J Thorac Cardiovasc Surg 1981; 81:921-7.

10. Shumacker Junior H, Lowenberg R. Experimental studies in vascular repair: comparision of various methods of end-toend arterial sutures. Surgery 1948; 24: 79-89.
11. Steen S, Anderson L, Lowenhielm P, Stridbeck H, Walther B, Holmin T. Comparison between absorbable and non absorbable monofilament sutures for end-to-end suture anastomoses in growing pigs. Surgery 1984; 95: 202-8.

12. Stillman RM, Sophie Z. Repair of growing vassels: contínuous absorbable or interrupted nonabsorbable suture? Arch Surg 1985; 120: 1281-83.

13. Tawes RL, Aberdeen E, Berry C. The growth of an aortic anastomosis: an experimental study in piglets. Surgery; 1968, 64: 1122-32.

14. Verschure I, Francois K, De Roose J, Berzsenyi G, Derom,F. Polydioxanone suture material in growing vascular anastomoses: experimental study. J Thorac Cardiovasc Surg 1985;90: 765-70.

Campos NLKL, Ueda AK, Moraes-Silva MA. Arterial suture with continuous and interrupted techniques, using polypropylene and polydioxanone threads: experimental study in rabbits. Acta Cir Bras [serial online] 2003 Sept-Oct;18(5). Available from URL: http://www.scielo.br/acb.

ABSTRACT - Purpose: To assess the arterial suture, comparing continuous and interrupted techniques, in abdominal aorta of growing rabbbits, using two types of suture material: Polypropylene 7-0 (nonabsorbable) and Polydioxanone 7-0 (absorbable). Methods: Groups: GI - Control, without arterial suture; GII - Polypropylene, Interrupted technique; GIII - Polipropilene, Continuous technique; GIV - Polydioxanone, Interrupted technique and GV - Polydioxanone, Continuous technique. Each group was subdivided in four Moments of Euthanasia , according with the number of days after surgery: $7,14,30$ and 60 days. The following itens were assessed: weight, arterial diameters and pulse, stenosis, thrombosis, tissue adherence at the suture line, aortography, suture material visibility, suture line healling visibility and microscopic study. Results: a) after 60 days, the suture line site had a significant growing in all groups; b) interrupted technique result less suture line stenosis in surgical act and in euthanasia c) the difference between groups showed in the microscopic study, was transitory, not pesisting after 60 days post surgery; Conclusion: Polydioxanone is better than polypropylene for suture in growing artery, not causing (or causing little) restriction to the growing of the suture line, even though using continuous technique.

KEY WORDS - Artery. Suture. Polypropylene. Polydioxanone.

Conflito de interesse: nenhum Fonte de financiamento: nenhuma

Correspondência:

Nelson Leonardo Kerdahi Leite de Campos

Departamento de Cirurgia e Ortopedia - Disciplina de Cirurgia Cardiovascular

Faculdade de Medicina de Botucatu - UNESP

R. Rubião Junior, s/n

18618970 Botucatu - SP

ncampos@fmb.unesp.br

Data do recebimento: $25 / 06 / 2003$

Data da revisão: 02/07/2003

Data da aprovação: 15/07/2003 